\title{
Calibration and Balancing of Multiple Tuned Mass Absorbers for Damping of Coupled Bending-Torsion Beam Vibrations
}

\author{
Hoffmeyer, David; Høgsberg, Jan
}

Published in:

Journal of Vibration and Acoustics

Link to article, DOI:

$10.1115 / 1.4046752$

Publication date:

2020

Document Version

Peer reviewed version

Link back to DTU Orbit

Citation (APA):

Hoffmeyer, D., \& Høgsberg, J. (2020). Calibration and Balancing of Multiple Tuned Mass Absorbers for Damping of Coupled Bending-Torsion Beam Vibrations. Journal of Vibration and Acoustics, 142(4), [044501].

https://doi.org/10.1115/1.4046752

\section{General rights}

Copyright and moral rights for the publications made accessible in the public portal are retained by the authors and/or other copyright owners and it is a condition of accessing publications that users recognise and abide by the legal requirements associated with these rights.

- Users may download and print one copy of any publication from the public portal for the purpose of private study or research.

- You may not further distribute the material or use it for any profit-making activity or commercial gain

- You may freely distribute the URL identifying the publication in the public portal

If you believe that this document breaches copyright please contact us providing details, and we will remove access to the work immediately and investigate your claim. 


\title{
Calibration and Balancing of Multiple Tuned Mass Absorbers for Damping of Coupled Bending-Torsion Beam Vibrations
}

\author{
David Hoffmeyer \\ PhD Student \\ Department of Mechanical Engineering \\ Technical University of Denmark \\ DK-2800, Kongens Lyngby, Denmark \\ Email: daho@iti.sdu.dk \\ Jan Høgsberg \\ Associate Professor \\ Technical University of Denmark \\ DK-2800, Kongens Lyngby, Denmark \\ Email: jhg@mek.dtu.dk
}

\begin{abstract}
For damping of fully coupled beam vibrations a threecomponent tuned mass absorber is proposed, consisting of two translational and a single rotational absorber, which together target a specific vibration mode. The representation of the interaction with residual non-resonant vibration modes is consistently included by two supplemental terms in the absorber equation, calibrated by frequency matching with the full structural model for vanishing absorber damping. The absorber tuning directly includes these residual terms without approximation when the underlying modal representation is based on the vibration form with absorber masses rigidly attached to the structure. It is demonstrated that the desired damping behaviour require non-homogeneous absorber balancing, obtained by an approximate method for the relative sizing of each absorber mass.
\end{abstract}

\section{Introduction}

Supplemental damping of flexible beam structures is conveniently introduced by tuned mass absorbers [1] working as suspended masses connected to the structure by springs and viscous dashpots. Such device configurations have been used for vibration mitigation of e.g. wind turbine blades [2], suspension bridges [3,4] and tall buildings [5].

Tuning of the mass absorber relies on accurate frequency matching with a selected vibration mode of the flexible host structure. The classic tuning procedure of Den Hartog [6] is based on attaining equal dynamic amplification at two neutral frequencies at which the amplitude is independent of the device damping. Specifically for tuned mass absorbers this principle also implies equal damping of the two modes associated with the selected vibration form of the structure [7].
Most tuning procedures assume that the response of the structure can be represented solely by the targeted vibration mode, although the absorber will inherently experience supplemental support motion from the other non-resonant modes. This interaction with residual vibration modes deteriorates the calibration, unless corrected by e.g. the addition of a flexibility and an inertia term in the absorber equation, as proposed in [8]. The present paper adopts a slightly alternative correction approach, in which the additional parameters are calibrated based on matching of the two frequencies associated with the resonant structural modes at vanishing absorber damping, as proposed for inerter-based absorbers in [9].

Multiple absorbers may be applied to damp coupled torsionbending vibrations in slender beam-like structures $[10,11]$. As many tall buildings have an irregular shape, multiple absorbers are furthermore used to mitigate response from seismic loading [12]. However, explicit procedures for the sizing and thereby relative balancing of the individual absorbers often rely on a numerical optimization procedure [13-15], which may be inconvenient with non-parametric solutions.

The present note proposes a three-component mass absorber for damping of fully coupled beam vibrations, consisting of a single rotational and two translational absorbers. These three absorbers may be placed at the same location on the beam, as they are associated with different structural degrees-offreedom. The configuration of the combined translationalrotational absorbers for coupled-mode vibration damping may be realized by a crucifix-type absorber [16] or a similar coupled beam-type absorber [17], both originally proposed for damping of pure bending and/or torsion modes 
with well-separated frequencies. For the present problem, in which the multiple absorber configuration targets a single coupled mode, the balancing between the individual absorber components becomes important. Although the method proposed in [18] has been derived for relative sizing of viscous dampers, it is directly translated to the present mass absorber problem, for which it appears to give a proper balancing of the three individual absorber components when targeting a mode with inherent bend-twist coupling.

The method uses a modal expansion with the infinitely damped mode shapes as basis. As a result, the governing characteristic equation is given without approximation. The design procedure assumes knowledge of the undamped frequencies of the structure with the absorbers attached. Thus, the advantage of the proposed method is that uncertainties in geometry, boundary conditions etc., impairing the calibration, are contained in these natural frequencies, readily measured for the actual structure, furthermore enabling absorber retuning during the structures life-time.

\section{Governing equations}

\subsection{Coupled differential beam equations}

For a beam structure with a cross-section that lacks double symmetry, bending and torsional vibrations inherently couple. With $z$ as axial coordinate through the shear centre, the transverse displacements $\left\{\xi_{1}, \xi_{2}\right\}$ and the angle of twist $\theta$ are governed by the coupled partial differential equations [19]

$$
\begin{array}{r}
E I_{\alpha \beta} \xi_{\beta}^{\prime \prime \prime \prime}+\rho A \ddot{\xi}_{\alpha}-\rho A e_{\alpha \beta} c_{\beta} \ddot{\theta}=f_{\alpha}^{e}-f_{\alpha}^{a} \delta\left(z-z_{a}\right) \\
E I_{\psi} \theta^{\prime \prime \prime \prime \prime}-G K \theta^{\prime \prime}+\rho A c_{\alpha} e_{\alpha \beta} \ddot{\xi}_{\beta}+\rho J \ddot{\theta}=f_{\theta}^{e}-f_{\theta}^{a} \delta\left(z-z_{a}\right)
\end{array}
$$

with implied summation over repeated Greek indices $\alpha, \beta=1,2$. The external forces are given by $f^{e}$, while Dirac's delta functions $\delta()$ specify the discrete location $z=z_{a}$ of the absorber forces $f^{a}$. The beam cross-section parameters are the bending stiffness $E I_{\alpha \beta}$, torsion rigidity $G K$, warping stiffness $E I_{\psi}$, mass per unit length $\rho A$ and torsional inertia per unit length $\rho J$. Spatial differentiation is indicated by ()$^{\prime}=\partial() / \partial z$, while ()$=\partial() / \partial t$ denotes differentiation with respect to time $t$. The coupling is explicitly given by the distances $c_{\alpha}$ between the shear and elastic centres, which vanishes for double cross-section symmetry and enters (1) by the permutation symbol $e_{\alpha \beta}$.

A discretized system of equations is conveniently obtained from (1) by applying the principle of virtual work. The three coupled equations (1) are therefore multiplied by their respective virtual functions $\widetilde{\xi}_{\alpha}$ and $\widetilde{\theta}$, integrated over the full length of the beam $\ell$ and then added to constitute a single weak form equation, in which the elastic terms are consistently integrated by parts to secure symmetric stiffness contributions and define boundary conditions and section forces. The two transverse displacements $\xi_{\alpha}=\mathbf{N}_{\alpha} \mathbf{q}$ and the angle of twist $\boldsymbol{\theta}=\mathbf{N}_{\theta} \mathbf{q}$ are then respectively interpolated by cubichermitian shape functions in $\mathbf{N}_{1}, \mathbf{N}_{2}$ and $\mathbf{N}_{\theta}$ with respect to the displacement vector $\mathbf{q}$ containing all degrees of freedom (dofs). The weak form equation can then be written as

$$
\widetilde{\mathbf{q}}(t)^{T}\left(\mathbf{K q}(t)+\mathbf{M} \ddot{\mathbf{q}}(t)-\mathbf{f}_{e}(t)+\mathbf{W} \mathbf{f}_{a}(t)\right)=0
$$

introducing the stiffness matrix (with summation over repeated index $\alpha=1,2$ )

$$
\begin{array}{r}
\mathbf{K}=\int_{0}^{\ell}\left(\left(\mathbf{N}_{\alpha}^{\prime \prime}\right)^{T} E I_{\alpha \beta} \mathbf{N}_{\alpha}^{\prime \prime}+\left(\mathbf{N}_{\theta}^{\prime \prime}\right)^{T} E I_{\psi} \mathbf{N}_{\theta}^{\prime \prime}\right. \\
\left.+\left(\mathbf{N}_{\theta}^{\prime}\right)^{T} G K \mathbf{N}_{\theta}^{\prime}\right) d z
\end{array}
$$

the mass matrix

$$
\begin{array}{r}
\mathbf{M}=\int_{0}^{\ell}\left(\rho A \left(\mathbf{N}_{\alpha}^{T} \mathbf{N}_{\alpha}-\mathbf{N}_{\alpha}^{T} e_{\alpha \beta} c_{\beta} \mathbf{N}_{\theta}\right.\right. \\
\left.+\mathbf{N}_{\theta}^{T} c_{\alpha} e_{\alpha \beta} \mathbf{N}_{\beta}\right) \\
\left.+\rho J \mathbf{N}_{\theta}^{T} \mathbf{N}_{\theta}\right) d z
\end{array}
$$

the external load vector

$$
\mathbf{f}_{e}=\int_{0}^{\ell}\left(\mathbf{N}_{\alpha}^{T} f_{\alpha}^{e}+\mathbf{N}_{\theta}^{T} f_{\theta}^{e}\right) d z
$$

and the absorber force vector $\mathbf{f}_{a}=\left[f_{1}^{a}, f_{2}^{a}, f_{\theta}^{a}\right]^{T}$ multiplied by the $n \times 3$ connectivity array

$$
\mathbf{W}=\left[\mathbf{N}_{1}^{T}, \mathbf{N}_{2}^{T}, \mathbf{N}_{\theta}^{T}\right]_{z=z_{a}}
$$

represented by the value of the three global shape function vectors at the absorber coordinate $z=z_{a}$. In practice the integrals in (3) to (5) are conveniently solved element-wise without explicit use of $\mathbf{N}_{1}, \mathbf{N}_{2}$ and $\mathbf{N}_{\theta}$, and subsequently placed in the global matrix/vector form by an assembly routine.

\subsection{Structure and absorber}

The governing discretized system of equations are obtained by vanishing of the large parenthesis in the weak form relation (2). When assuming a harmonic time representation $\mathbf{q}(t)=\mathbf{q} e^{i \omega t}$ and $\mathbf{f}(t)=\mathbf{f} e^{i \omega t}$, with $\omega$ being the angular frequency, the frequency response of the beam structure is governed by the equation of motion

$$
\left(\mathbf{K}-\omega^{2} \mathbf{M}\right) \mathbf{q}+\mathbf{W} \mathbf{f}_{a}=\mathbf{f}_{e}
$$

Although equation (1) indicates $n_{a}=3$ tuned mass absorbers, the format of the discretized system is generic and therefore easily extended to additional absorbers with inhomogeneous 
(a)

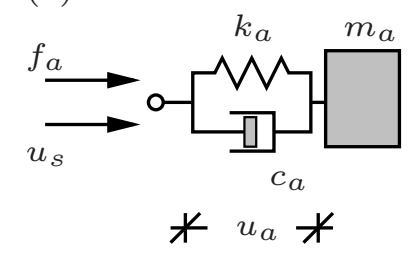

(b)

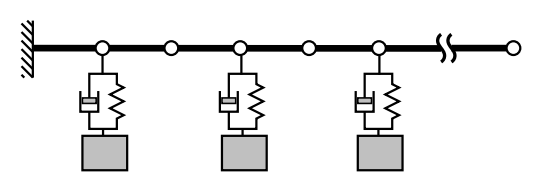

(c)

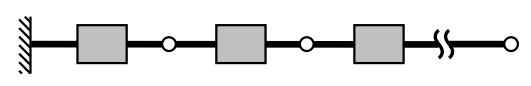

Fig. 1. (a) Tuned mass absorber, (b) structure with multiple absorbers and (c) locking of the absorber mass to the structure at $c_{a} \rightarrow \infty$.

locations along the beam. The three absorber forces are collected in the vector $\mathbf{f}_{a}$ with the connectivity array given by

$$
\mathbf{W}=\left[\mathbf{w}_{1}, \mathbf{w}_{2}, \mathbf{w}_{\theta}\right]
$$

specifying the attachment of each absorber on the structure and thus defining

$$
\mathbf{u}_{s}=\mathbf{W}^{T} \mathbf{q}
$$

as a vector containing the structural base motion experienced by each absorber (see Fig. 1a). In the present analysis any inherent structural damping is neglected. However, as shown in [20], the absorbers may be calibrated accurately without considering the structural damping.

Figure 1a shows a single absorber comprising a mass $m_{a}$ connected to the structure by a spring with stiffness $k_{a}$ in parallel with a viscous dashpot with coefficient $c_{a}$. The absorber forces $\mathbf{f}_{a}$ can thus be determined as

$$
\mathbf{f}_{a}=-\left(k_{a}+i \omega c_{a}\right) \boldsymbol{\alpha} \mathbf{u}_{a}=-\omega^{2} m_{a} \boldsymbol{\alpha}\left(\mathbf{u}_{a}+\mathbf{u}_{s}\right)
$$

where the vector $\mathbf{u}_{a}$ contains the relative displacements of the absorber masses, while the relative sizing of the absorbers in Fig. $1 \mathrm{~b}$ is given by the balancing matrix

$$
\boldsymbol{\alpha}=\left[\begin{array}{lll}
\alpha_{1} & & \\
& \alpha_{2} & \\
& & \alpha_{\theta}
\end{array}\right]
$$

treated in further detail in Section 6.

When introducing $\mathbf{u}_{s}=\mathbf{W}^{T} \mathbf{q}$, the latter equality in (10) gives

$$
\left(k_{a}+i \omega c_{a}-\omega^{2} m_{a}\right) \boldsymbol{\alpha} \mathbf{u}_{a}-\omega^{2} m_{a} \boldsymbol{\alpha} \mathbf{W}^{T} \mathbf{q}=\mathbf{0}
$$

with common absorber parameters $m_{a}, c_{a}$ and $k_{a}$ for a given balancing matrix $\boldsymbol{\alpha}$.

The corresponding structural equation (7) is obtained by elimination of the absorber force $\mathbf{f}_{a}$ given by the latter expression in (10). Hereby the structural equation of motion can be written as

$$
\left(\mathbf{K}-\omega^{2} \mathbf{M}_{\infty}\right) \mathbf{q}-\omega^{2} m_{a} \mathbf{W} \boldsymbol{\alpha} \mathbf{u}_{a}=\mathbf{f}_{e}
$$

with the augmented mass matrix

$$
\mathbf{M}_{\infty}=\mathbf{M}+m_{a} \mathbf{W} \boldsymbol{\alpha} \mathbf{W}^{T}
$$

containing contributions from the three absorber masses, as illustrated in Fig. 1c.

\subsection{Modal properties}

Without absorbers and external load the original structural equation (7) defines the undamped eigenvalue problem

$$
\left(\mathbf{K}-\omega_{0, j}^{2} \mathbf{M}\right) \mathbf{q}_{0, j}=\mathbf{0}
$$

in which the eigenvalue $\omega_{0, j}$ is the undamped natural frequency of mode $j$ and $\mathbf{q}_{0, j}$ is corresponding mode shape vector. Although commonly used for a modal representation of the structure to be damped, it may appear inconsistent, as (15) is not recovered in either of the limits $c_{a} \rightarrow 0$ or $\infty$ for the absorber damping coefficient.

The alternative equation of motion (13) recovers the undamped structure in Fig. 1c for $c_{a} \rightarrow \infty$, with all absorber masses included in the mass matrix (14). Therefore, the corresponding eigenvalue problem

$$
\left(\mathbf{K}-\omega_{\infty, j}^{2} \mathbf{M}_{\infty}\right) \mathbf{q}_{\infty, j}=\mathbf{0}
$$

serves as a consistent basis for a modal truncation of the flexible structure. The eigenvalue of (16) determines $\omega_{\infty, j}<\omega_{0, j}$ as the infinitely damped natural frequency, while $\mathbf{q}_{\infty, j}$ is the corresponding mode shape vector.

All absorbers are calibrated towards a single resonant vibration mode $j=r$. With the alternative problem (16) as basis, the modal expansions can be expressed as

$$
\mathbf{q}=\sum_{j=1}^{n} \mathbf{q}_{\infty, j} \frac{x_{j}}{v_{\infty, j}} \quad, \quad \mathbf{u}_{a}=\mathbf{W}^{T} \mathbf{q}_{\infty, r} \frac{y_{r}}{v_{\infty, r}}
$$

with $x_{j}$ and $y_{r}$ being the modal coordinates for the structure and absorber displacements, respectively. The collective absorber coordinate $y_{r}$ for the target mode $j=r$ may without loss of generality be expressed directly in terms of $\mathbf{q}_{\infty, r}$, providing an identical coupling coefficient in the subsequent 
modal equations. A combined modal amplitude $v_{\infty, j}$ at the absorber locations for a specific mode $j$ is in (17) introduced by the quadratic relation

$$
v_{\infty, j}^{2}=\mathbf{q}_{\infty, j}^{T} \mathbf{W} \boldsymbol{\alpha} \mathbf{W}^{T} \mathbf{q}_{\infty, j}
$$

comprising the location of the absorbers via $\mathbf{W}$ and the relative sizing through the balancing matrix $\boldsymbol{\alpha}$.

When introducing the expansion in (17) into the structural equation in (13) and then pre-multiplying with $\mathbf{q}_{\infty, j}^{T} / \mathbf{v}_{\infty, j}$, the corresponding modal equation for a mode $j$ appears in uncoupled form as

$$
\left(k_{j}-\omega^{2} m_{j}\right) x_{j}-\omega^{2} m_{a} \eta_{j r} y_{r}=f_{j}
$$

defining the structural modal mass, modal stiffness and external modal load as

$$
m_{j}=\frac{\mathbf{q}_{\infty, j}^{T} \mathbf{M}_{\infty} \mathbf{q}_{\infty, j}}{v_{\infty, j}^{2}} \quad, \quad k_{j}=\frac{\mathbf{q}_{\infty, j}^{T} \mathbf{K} \mathbf{q}_{\infty, j}}{v_{\infty, j}^{2}} \quad, \quad f_{j}=\frac{\mathbf{q}_{\infty, j}^{T} \mathbf{f}_{e}}{v_{\infty, j}}
$$

whereby the infinitely damped natural frequency $\omega_{\infty, j}$ is defined by the modal stiffness to mass ratio $\omega_{\infty, j}^{2}=k_{j} / m_{j}$. The normalized modal absorber displacement $y_{r}$ in (17b) introduces the modal coupling coefficient

$$
\eta_{j r}=\frac{\mathbf{q}_{\infty, j}^{T} \mathbf{W} \boldsymbol{\alpha} \mathbf{W}^{T} \mathbf{q}_{\infty, r}}{\boldsymbol{v}_{\infty, j} \mathbf{v}_{\infty, r}}
$$

in (19) so that $\eta_{r r}=1$. By pre-multiplication of (12) with $\mathbf{q}_{\infty, r}^{T} \mathbf{W} / \mathrm{v}_{\infty, r}$, the associated modal absorber equation is given as

$$
\left(k_{a}+i \omega c_{a}-\omega^{2} m_{a}\right) y_{r}-\omega^{2} m_{a} \sum_{j=1}^{n} \eta_{r j} x_{j}=0
$$

with the sum containing contributions from all vibration modes through the coupling coefficient $\eta_{r j}$ in (21). In the coupled modal equations (19) and (22) the $n_{a}=3$ tuned mass absorbers act as a single unit with their individual balancing defined by the matrix $\boldsymbol{\alpha}$, while the sum with $\eta_{r j}$ contains the background contributions from residual vibration modes $j \neq r$.

\subsection{Modal representation with residual mode correc- tion}

Instead of truncating the sum in (22) to a single term $j=r$, the residual terms $j \neq r$ may be included by correction terms, as suggested in [8]. It follows from the modal structural equation (19) that $x_{j}$ is proportional to $y_{r}$ for free vibrations. Thus, the residual components of the sum in (22) may be represented consistently by a flexibility and an inertia correction term that must be proportional to $y_{r}$,

$$
\sum_{j=1}^{n} \eta_{r j} x_{j}=x_{r}+\sum_{j \neq r}^{n} \eta_{r j} x_{j}=x_{r}+k_{a}\left(\frac{1}{k_{r}^{\prime}}-\frac{1}{m_{r}^{\prime} \omega^{2}}\right) y_{r}
$$

The correction terms are directly introduced by a supplemental modal stiffness $k_{r}^{\prime}$ and modal mass $m_{r}^{\prime}$, representing base motion flexibility parameters via the sum of their reciprocal values. In [8] the supplemental correction parameters are determined for a single absorber in an explicit way based on the system matrices. The paper furthermore adopts a similar criterion for the tuning procedure as in the following Section 3 but only assumes a single absorber. As will be shown, the current approach instead determines these parameters by matching with frequencies that could be obtained from actual measurements or dedicated numerical analysis.

The modal truncation (23) with residual mode correction eliminates the sum in the absorber equation (22), whereafter the two terms with $k_{r}^{\prime}$ and $m_{r}^{\prime}$ are fully absorbed by the corresponding physical terms proportional to $y_{r}$.

For the structural modal equation (19) the specific equation for the targeted vibration mode $j=r$ can now be normalized by the modal stiffness $k_{r}$ as

$$
\left(1-\xi^{2}\right) x_{r}-\mu_{r} \xi^{2} y_{r}=0
$$

introducing the non-dimensional frequency

$$
\xi=\frac{\omega}{\omega_{\infty, r}}=\omega \sqrt{\frac{m_{r}}{k_{r}}}
$$

Similarly the absorber equation (22), with the sum eliminated by (23), is normalized by division with $k_{r}$. Hereby, it can be written in compact form as

$$
\left(\kappa_{*}+i \xi \beta_{*}-\xi^{2} \mu_{*}\right) y_{r}-\xi^{2} \mu_{*} \kappa_{*} \frac{1}{\kappa_{r}} x_{r}=0
$$

where the corrected mass, stiffness and damper ratios with asterisks

$$
\frac{1}{\mu_{*}}=\frac{1}{\mu_{r}}+\frac{1}{\mu_{r}^{\prime}} \quad, \quad \frac{1}{\kappa_{*}}=\frac{1}{\kappa_{r}}+\frac{1}{\kappa_{r}^{\prime}} \quad, \quad \beta_{*}=\beta_{r} \frac{\kappa_{*}}{\kappa_{r}} \frac{\mu_{*}}{\mu_{r}}
$$

are expressed by the physical absorber mass, stiffness and damper ratios

$$
\mu_{r}=\frac{m_{a}}{m_{r}} \quad, \quad \kappa_{r}=\frac{k_{a}}{k_{r}} \quad, \quad \beta_{r}=\frac{c_{a}}{\sqrt{k_{r} m_{r}}}
$$

and the corrected mass and stiffness ratios with primes

$$
\mu_{r}^{\prime}=\frac{m_{r}^{\prime}}{m_{r}} \quad, \quad \kappa_{r}^{\prime}=\frac{k_{r}^{\prime}}{k_{r}}
$$


representing the residual mode contributions introduced in (23). Because of the modal expansion with the infinitely damped mode shapes $\mathbf{q}_{\infty, j}$, the residual correction terms are fully included in the comprised ratios $\mu_{*}$ and $\kappa_{*}$ without any approximations, as otherwise needed for tuned vibration absorbers when the undamped mode shapes $\mathbf{q}_{0, j}$ are used in the modal decomposition $[8,21]$.

\section{Absorber tuning}

The tuning of the absorbers is based on the properties of the complex poles, governed by the characteristic equation obtained by eliminating the modal coordinates between the two equations (26) and (24). By re-arranging terms the characteristic equation can be written as

$$
\mu_{*}\left(1-\kappa_{*} \frac{\mu_{r}}{\kappa_{r}}\right) \xi^{4}-\left(\mu_{*}+\kappa_{*}\right) \xi^{2}+\kappa_{*}+i \xi \beta_{*}\left(1-\xi^{2}\right)=0
$$

which remains within a fourth-order polynomial format only because the structural equation (13) contains the augmented mass matrix $\mathbf{M}_{\infty}$ defined in (14).

The system parameters in (30) are determined based on a specific tuning criterion, which in the present case is the equal modal concept described in [7, 22]. For tuned mass absorbers it secures a good compromise between large attainable damping and effective response amplitude reduction for harmonic excitation of the structure. In case of e.g. base excitation, the equal damping property will secure effective mitigation, although the amplitude reduction will only be suboptimal.

The procedure relies on the exact matching of the characteristic equation (30) with the generic polynomial

$$
\xi^{4}-\left(2+4 \chi^{2}\right) \xi_{0}^{2} \xi^{2}+\xi_{0}^{4}+4 i \lambda \chi \xi_{0} \xi\left(\xi_{0}^{2}-\xi^{2}\right)=0
$$

which by construction secures equal damping and obtains maximum damping ratio at a bifurcation point for $\lambda=1$. It follows immediately by the ratio between the first and third order terms inside the last parenthesis of (30) and (31) that the reference frequency ratio

$$
\xi_{0}=1
$$

which is a direct consequence of using the locked mode shapes $\mathbf{q}_{\infty, j}$ as the basis of the modal representation. Next the characteristic equation (30) is normalized to a unit coefficient to the leading quartic term. Then comparison of the constant terms in (30) and (31) gives

$$
\frac{\kappa_{*}}{\mu_{*}}=\frac{1}{1+\mu_{*} \frac{\mu_{r}}{\kappa_{r}}}
$$

identifying $\mu_{*} \mu_{r} / \kappa_{r}$ as a representative absorber parameter. Comparison of the quadratic terms gives the model parame- ter

$$
\chi=\sqrt{\frac{1}{4} \mu_{*} \frac{\mu_{r}}{\kappa_{r}}}
$$

while the corrected damper ratio $\beta_{*}$ is then determined from the common factors to the odd-power terms,

$$
\frac{\beta_{*}}{\kappa_{*}}=\sqrt{2 \mu_{*} \frac{\mu_{r}}{\kappa_{r}}}
$$

In this expression for $\beta_{*}$ a complex pole location slightly below the bifurcation point is achieved by $\lambda=\frac{1}{2} \sqrt{2}$, which avoids structural interference of the two identical modes at $\lambda=1$ and thereby provides the desired flat plateau in the frequency response curves [7].

Finally, the attainable modal damping ratio in the two structural modes may be estimated from (34) as [22]

$$
\zeta_{r}^{\text {est }} \simeq \lambda \chi=\sqrt{\frac{1}{8} \mu_{*} \frac{\mu_{r}}{\kappa_{r}}}
$$

The above expressions ensure equal modal damping of the two structural modes based on a specified mass ratio $\mu_{r}$. Furthermore, when the corrections for residual modes are not included, the expressions (33)-(36) simplify as $\mu_{*}=\mu_{r}$ and $\kappa_{*}=\kappa_{r}$.

\section{Calibration of residual mode parameters}

In (23) the correction modal stiffness $k_{r}^{\prime}$ and mass $m_{r}^{\prime}$ represent the influence by all other vibration modes and thus explicitly adjust the actual system parameters $\mu_{r}$ and $\kappa_{r}$ in (27) by their corresponding ratios $\kappa_{r}^{\prime}$ and $\mu_{r}^{\prime}$ defined in (29). The influence of other modes on the targeted vibration mode may be assessed in the limit $c_{a}=0$, in which the homogeneous form of the governing equations (13) and (12) determine $n_{a}+1$ natural frequencies for the vibration mode $j=r$ when the absorber parameters $m_{a}$ and $k_{a}$ are calibrated according to the tuning principle of Section 3.

Assume that the absorber mass and stiffness can be estimated as $m_{a}=\widetilde{m}_{a}$ and $k_{a}=\widetilde{k}_{a}$. For $c_{a}=0$ the homogeneous form of (13) and (12) then constitute the eigenvalue problem

$$
\left(-\omega^{2}\left[\begin{array}{cc}
\mathbf{M}+\widetilde{m}_{a} \mathbf{W} \boldsymbol{\alpha} \mathbf{W}^{T} & \widetilde{m}_{a} \mathbf{W} \boldsymbol{\alpha} \\
\widetilde{m}_{a} \boldsymbol{\alpha} \mathbf{W}^{T} & \widetilde{m}_{a} \boldsymbol{\alpha}
\end{array}\right]+\left[\begin{array}{cc}
\mathbf{K} & \mathbf{0} \\
\mathbf{0}^{T} & \widetilde{k}_{a} \boldsymbol{\alpha}
\end{array}\right]\right)\left[\begin{array}{c}
\mathbf{q} \\
\mathbf{u}_{a}
\end{array}\right]=\underset{(37)}{\left[\begin{array}{l}
\mathbf{0} \\
\mathbf{0}
\end{array}\right]}
$$

When $\widetilde{m}_{a}$ and $\widetilde{k}_{a}$ are chosen sufficiently accurate, this eigenvalue problem (37) identifies $n_{a}+1$ natural frequencies around $\omega_{\infty, r}$ for the targeted vibration mode $r$. Two of these frequencies $\omega_{A}$ and $\omega_{B}$ represent dynamic absorber-structure interaction of the actual flexible structure, while the remaining $n_{a}-1$ frequencies describe redundant absorber modes with high damping. Figure 3 shows trajectories with respect to $c_{a}$ of the $n_{a}+1=4$ natural frequencies in the complex 
plane for the numerical example considered in Section 7. For $c_{a}=0$ the associated four real-valued frequencies are then identified in Fig. 3c, with the two outer frequencies identified or assumed as $\omega_{A}(\circ)$ and $\omega_{B}(\square)$, while the two intermediate frequencies $(\times)$ then denote the redundant trajectories that approach the imaginary axis for increasing $c_{a}$.

The background correction ratios $\kappa_{r}^{\prime}$ and $\mu_{r}^{\prime}$ are now determined such that the characteristic equation (30) exactly recovers the determined frequency ratios

$$
\xi_{A, B}=\frac{\omega_{A, B}}{\omega_{\infty, r}}
$$

in the limit $\beta_{*}=0$, where (30) reduces to the quadratic equation

$$
\widetilde{\mu}_{*}\left(1-\widetilde{\boldsymbol{\kappa}}_{*} \frac{\widetilde{\boldsymbol{\mu}}_{r}}{\widetilde{\kappa}_{r}}\right) \xi^{4}-\left(\widetilde{\mu}_{*}+\widetilde{\boldsymbol{\kappa}}_{*}\right) \xi^{2}+\widetilde{\boldsymbol{\kappa}}_{*}=0
$$

with assumed mass and stiffness ratios $\widetilde{\mu}_{r}=\widetilde{m}_{a} / m_{r}$ and $\widetilde{\kappa}_{r}=$ $\widetilde{k}_{a} / k_{r}$, respectively. The sum and product of the two roots $\xi_{A}^{2}$ and $\xi_{B}^{2}$ to (39) then fulfill the relations

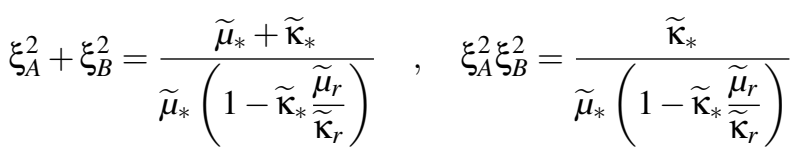

from which the combined stiffness and mass ratios are readily obtained as

$$
\begin{aligned}
\widetilde{\mu}_{*} & =\frac{\widetilde{\kappa}_{r}}{\widetilde{\mu}_{r}} \frac{\xi_{A}^{2} \xi_{B}^{2}-\xi_{A}^{2}-\xi_{B}^{2}+1}{\xi_{A}^{2} \xi_{B}^{2}-\xi_{A}^{2}-\xi_{B}^{2}} \\
\widetilde{\kappa}_{*} & =-\frac{\widetilde{\kappa}_{r}}{\widetilde{\mu}_{r}} \frac{\xi_{A}^{2} \xi_{B}^{2}-\xi_{A}^{2}-\xi_{B}^{2}+1}{\xi_{A}^{2} \xi_{B}^{2}}
\end{aligned}
$$

Finally, the correction ratios $\mu_{r}^{\prime}$ and $\kappa_{r}^{\prime}$ are determined from (27) as

$$
\frac{1}{\mu_{r}^{\prime}}=\frac{1}{\widetilde{\mu}_{*}}-\frac{1}{\widetilde{\mu}_{r}} \quad, \quad \frac{1}{\kappa_{r}^{\prime}}=\frac{1}{\widetilde{\kappa}_{*}}-\frac{1}{\widetilde{\kappa}_{r}}
$$

As shown in the numerical example of Section 7 this procedure provides an accurate estimation of the residual mode correction ratios $\kappa_{r}^{\prime}$ and $\mu_{r}^{\prime}$, provided that the initial estimates $\widetilde{m}_{a}$ and $\widetilde{k}_{a}$ are sufficiently accurate and that $\omega_{A}$ and $\omega_{B}$ can be clearly identified from the $n_{a}+1$ natural frequencies obtained for $c_{a}=0$.

\section{Design procedure}

A design procedure is now proposed and stepwise summarized in Table 1. The attainable damping ratio for mode $r$ can be estimated by (36). Without residual mode correction $\mu_{*}=\mu_{r}$ and $\kappa_{*}=\kappa_{r}$, whereby (33) determines $\kappa_{r}=$ $\mu_{r}\left(1-\mu_{r}\right)$ and (36) then gives a desired modal mass ratio

$$
\mu_{\mathrm{des}}=\frac{8 \zeta_{\mathrm{des}}^{2}}{1-8 \zeta_{\mathrm{des}}^{2}}
$$

when $\zeta_{r}^{\text {est }}=\zeta_{\text {des }}$ has been introduced. This mass ratio $\mu_{\mathrm{des}}$ can be used to determine a desired absorber mass $m_{a}$. However, the absorber ratios are defined in (28) based on the modal parameters with respect to the infinitely damped mode shape $\mathbf{q}_{\infty, r}$, for which the corresponding mass matrix $\mathbf{M}_{\infty}$ contains the unknown absorber mass. Thus, to estimate the desired absorber mass, the approximation $\mathbf{q}_{\infty, r} \simeq \mathbf{q}_{0, r}$ is substituted into (20a) with $\mathbf{M}_{\infty}$ eliminated by (14). Hereby the absorber mass is given as

$$
m_{a}=m_{0, r} \frac{\mu_{\mathrm{des}}}{1-\mu_{\mathrm{des}}}
$$

where $m_{0, r}$ is the mass ratio for the undamped structure obtained by (20a) with $\mathbf{q}_{\infty, r}=\mathbf{q}_{0, r}$ and $\mathbf{M}_{\infty}=\mathbf{M}$. The absorber mass $m_{a}$ is kept constant during the remainder of the design procedure, whereby $\mathbf{M}_{\infty}$ can be constructed by (14) and the infinitely damped eigenvalue problem (16) can then be solved to give $\omega_{\infty, r}$ and $\mathbf{q}_{\infty, r}$ for the target mode $j=r$. Hereby, the modal mass $m_{r}$ and stiffness $k_{r}$ are determined by (20), while the modal mass ratio is finally determined as $\mu_{r}=m_{a} / m_{r}$.

The absorber tuning in Section 3 requires calibration of the residual mode correction ratios $\mu_{r}^{\prime}$ and $\kappa_{r}^{\prime}$, obtained by the frequency matching procedure described in Section 4. Initially the stiffness ratio is estimated from (33) without correction and thus $\kappa_{*}=\kappa_{r}$, whereby the absorber mass and stiffness estimates are

$$
\tilde{m}_{a}=m_{a} \quad, \quad \widetilde{k}_{a}=k_{r} \mu_{r}\left(1-\mu_{r}\right)
$$

The augmented eigenvalue problem in (37) is now solved and the natural frequencies $\omega_{A, B}$ are identified among the $n_{a}+$ 1 natural frequencies associated with the vibration mode $r$, whereby the normalized frequencies are $\xi_{A, B}=\omega_{A, B} / \omega_{\infty, r}$ from (38). Thus, the residual mode correction ratios $\mu_{r}^{\prime}$ and $\kappa_{r}^{\prime}$ are estimated explicitly by the expressions in (41) and (42) with estimated mass ratio $\widetilde{\mu}_{r}=\widetilde{m}_{a} / m_{r}=\mu_{r}$ and stiffness ratio $\widetilde{\kappa}_{r}=\widetilde{k}_{a} / k_{r}=\mu_{r}\left(1-\mu_{r}\right)$ from $(45)$.

The obtained correction ratios $\mu_{r}^{\prime}$ and $\kappa_{r}^{\prime}$ represent the modal interaction with the non-resonant modes $j \neq r$, and thus the optimal absorber parameters must be re-tuned by the tuning expressions in Section 3. Because the absorber mass $m_{a}$ is not re-evaluated, the mass ratio $\mu_{r}$ may not be updated by (41a). An expression for the actual stiffness ratio $\kappa_{r}=k_{a} / k_{r}$ is therefore obtained by elimination of $\kappa_{*}$ between (27b) and the tuning relation (33), which gives

$$
\kappa_{r}=\frac{\mu_{*}\left(1-\mu_{r}\right)}{1-\mu_{*} / \kappa_{r}^{\prime}}
$$


Table 1. Design procedure for the tuned mass absorbers with $\zeta_{\text {des }}$.

1) Solve the undamped eigenvalue problem (15).

2) Determine the modal mass $m_{0, r}$.

3) Calculate the actual absorber mass $m_{a}$ by (44) and set $\widetilde{m}_{a}=m_{a}$.

4) Solve the infinitely damped eigenvalue problem (16).

5) Determine the modal mass $m_{r}$ and stiffness $k_{r}$ from (20).

6) Determine $\widetilde{m}_{a}$ and $\widetilde{k}_{a}$ by (45)

7) Solve the augmented eigenvalue problem (37) and choose $\omega_{A, B}$.

8) Determine $\mu^{\prime}$ and $\kappa^{\prime}$ from (41) and (42).

9) Re-evaluate $\kappa_{r}$ by (46) and $\kappa_{*}$ by (27b).

10) Evaluate $\beta_{*}$ by (35) and $\beta_{r}$ by (27c).

11) Determined actual absorber stiffness by $k_{a}=\kappa_{r} k_{r}$ and absorber damping coefficient by $c_{a}=\beta_{r} \sqrt{k_{r} m_{r}}$.

Without residual mode correction $\left(\kappa_{r}^{\prime} \rightarrow \infty\right)$ this expression recovers the correct solution $\kappa_{r}=\mu_{r}\left(1-\mu_{r}\right)$, used in (45b) to estimate the absorber stiffness. After determining $\kappa_{r}$ by (46) the combined stiffness ratio $\kappa_{*}$ is then finally re-calculated by (27b). As by now $\mu_{r}, \kappa_{r}, \mu_{*}$ and $\kappa_{*}$ are known, the combined damper ratio $\beta_{*}$ follows from the tuning formula (35), while the actual damper ratio $\beta_{r}$ is obtained from (27c). The absorber mass $m_{a}$ is initially chosen by (45), while the determination of the absorber stiffness $k_{a}=\mu_{r} k_{r}$ and damping coefficient $c_{a}=\beta_{r} \sqrt{k_{r} m_{r}}$ finalizes the design procedure, summarized in Table 1.

\section{Absorber balancing}

When multiple absorbers are placed on the same structure to damp the same mode, proper balancing factors should be introduced to relatively size the individual absorbers. In the force relation (10) the common absorber parameters $m_{a}$, $c_{a}$ and $k_{a}$ determine the magnitude for all absorbers, while their internal scaling is governed by the balancing matrix in (11).

The absorber force vector $\mathbf{f}_{a}$ in (10) can be expressed by an equivalent viscous relation as

$$
\mathbf{f}_{a}=i \omega H(\omega) \boldsymbol{\alpha} \mathbf{u}_{s}
$$

introducing the frequency dependent absorber function

$$
H(\omega)=m_{a} \frac{i \omega\left(k_{a}+i \omega c_{a}\right)}{-\omega^{2} m_{a}+k_{a}+i \omega c_{a}}
$$

which becomes real-valued for pure viscous dampers, as considered in [18]. An estimate of the absorber's working behavior can be gained by assuming resonance, where $m_{a} \omega^{2} \simeq k_{a}$, whereby the inertia and stiffness terms in the denominator of $H(\omega)$ cancel. Thus, the absorber function can be approximated as

$$
H(\omega) \simeq m_{a} \frac{i \omega\left(k_{a}+i \omega c_{a}\right)}{i \omega c_{a}} \simeq m_{a} \frac{k_{a}}{c_{a}}
$$

where the last approximation assumes $k_{a} \gg \omega c_{a}$, corresponding to $\sqrt{2 \mu_{r}} \ll 1$ for the absorber tuning assumed in Section 3 without residual mode correction. Thus, around a resonance the tuned mass absorber approximately acts as a viscous damper with apparent viscous coefficient $m_{a} k_{a} / c_{a}$. Thus, it seems appropriate for resonant absorber damping to determine a relative sizing of the individual absorbers by the approach for viscous dampers presented in [18].

Assume that each absorber acts directly on the structure, as indicated in Fig 1, whereby the connectivity vector for the $i$ 'th absorber

$$
\mathbf{w}_{i}=[0 \ldots, 1, \ldots, 0]^{T}
$$

contains a single unit value at the degree-of-freedom to which the $i$ 'th absorber is attached. Thus, $u_{s, i}=\mathbf{w}_{i}^{T} \mathbf{q}$ defines the $i$ 'th entry of $\mathbf{u}_{s}$ defined in (9). The balancing procedure in [18] secures that the viscous dampers restrain the displacement of the structure at the same rate, whereby they operate together optimally. The locked vibration mode, corresponding to having support conditions at the individual points of damper/absorber attachment can mathematically be formulated by introducing $n_{a}$ Lagrange multipliers in the extended eigenvalue problem [23],

$$
\left(\left[\begin{array}{cc}
\mathbf{K} & \mathbf{W} \\
\mathbf{W}^{T} & \mathbf{0}
\end{array}\right]-\overline{\boldsymbol{\omega}}_{\infty, r}^{2}\left[\begin{array}{cc}
\mathbf{M} & \mathbf{0} \\
\mathbf{0} & \mathbf{0}
\end{array}\right]\right)\left[\begin{array}{c}
\overline{\mathbf{q}}_{\infty, r} \\
\boldsymbol{\lambda}_{r}
\end{array}\right]=\left[\begin{array}{l}
\mathbf{0} \\
\mathbf{0}
\end{array}\right]
$$

where the $(\overline{)}$ refers to this eigenvalue problem with fully restrained structure displacements at all absorber locations. 
(a)

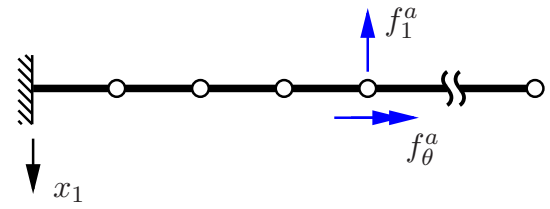

(b)

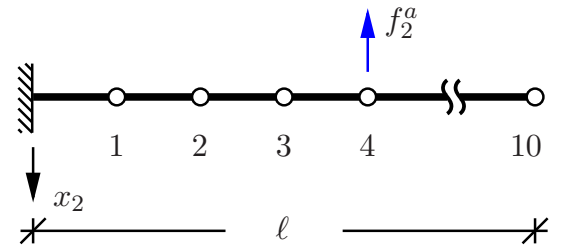

(c)

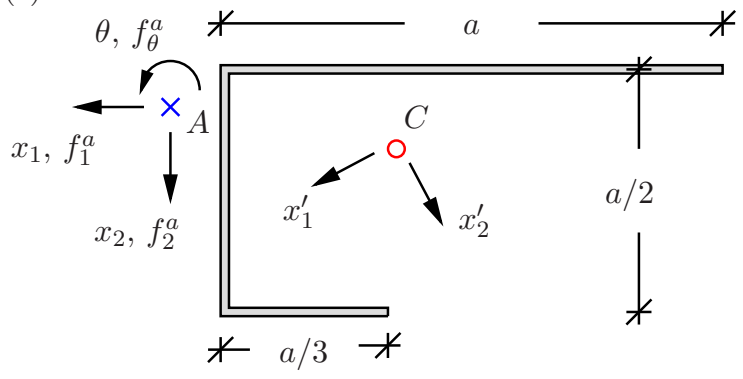

Fig. 2. (a,b) Cantilever beam with three absorber forces and (c) beam cross-section.

However, in practice the natural frequency $\bar{\omega}_{\infty, r}$ and mode shape $\overline{\mathbf{q}}_{\infty, r}$ are conveniently determined from the undamped eigenvalue problem (15) with the additional support conditions that restrain structural motion at all absorber locations. According to [18] the appropriate balancing of an absorber $i$ relative to a given reference $i=1$ is then obtained by the scaling relation

$$
\frac{\alpha_{i}}{\alpha_{1}}=\frac{\rho_{i, r} \mathbf{w}_{1}^{T} \mathbf{q}_{0, r}}{\rho_{1, r} \mathbf{w}_{i}^{T} \mathbf{q}_{0, r}}
$$

in which $\mathbf{q}_{0, r}$ is the undamped vibration mode $j=r$ from (15). The constraint force components $\rho_{i, r}$ for the $i$ 'th absorber in vibration mode $r$ are obtained by substitution of $\overline{\mathbf{q}}_{\infty, r}$ and $\bar{\omega}_{\infty, r}$ into the undamped balance equation (15) followed by pre-multiplication with $\mathbf{w}_{i}^{T}$ to extract the reaction force needed to restrain the structure,

$$
\rho_{i, r}=\mathbf{w}_{i}^{T}\left(\bar{\omega}_{\infty, r}^{2} \mathbf{M}-\mathbf{K}\right) \overline{\mathbf{q}}_{\infty, r}
$$

Note that the reaction force component may alternatively be extracted from (51) as the Lagrange multipliers in $\boldsymbol{\lambda}_{r}$. The balancing relation given by (52) ensures a uniform effect of the absorbers when working optimally together. Though the method has been formulated for viscous dampers in [18], quite good balancing factors are obtained for tuned mass absorbers, as illustrated by the following numerical example.

\section{Numerical example}

The calibration and balancing procedure is now illustrated by an example. The first mode of the cantilevered beam with length $\ell / a=40$ in Fig. $2 \mathrm{a}, \mathrm{b}$ is damped by three tuned mass absorbers. The beam cross-section is shown in Fig. 2c with indicated shear centre $A$ and elastic centre $C$. The axes $\left\{x_{1}^{\prime}, x_{2}^{\prime}\right\}$ indicate the principle axes and as the two centres do not coincide the translations in the $x_{1}$ - and $x_{2}$ directions couple with the angle of twist $\theta$. The cross-section geometry is given in the figure with overall thick $a / 40$. The material is isotropic with elastic modulus $E=210 \mathrm{GPa}$ and Poisson's ratio $v=0.3$. The beam is modelled by threedimensional beam elements with six degrees-of-freedom per node, including the rate of twist $\theta^{\prime}$. The beam model is discretized by ten elements and the absorbers $f_{1}^{a}, f_{2}^{a}$ and $f_{\theta}^{a}$ are placed in node four, as shown in Fig. 2a,b, with respect to the cross section shear centre $A$, as depicted in Fig. 2c. The attachment in the origin of the beam axes secures the single-unit-value construction of the connectivity vector $\mathbf{w}$ in (50), regarded by the balancing procedure in Section 6. In the present case the origin is placed in $A$ to obtain a simple structural model. For another absorber location, the beam axes must simply be placed at the different location.

For the approximate balancing procedure of Section 6, the eigenvalue problem (51) with constraints at absorber location determines the locked vibration mode $\overline{\mathbf{q}}_{\infty, 1}$ and natural frequency $\bar{\omega}_{\infty, 1}$ for the target mode $r=1$. The three constraint forces $\rho_{1,1}$ to $\rho_{3,1}$ are then determined by (53), while the components of the balancing matrix are finally evaluated by (52) as $\boldsymbol{\alpha}=\operatorname{diag}\left[1,1.735,0.038 a^{2}\right]$. This balancing ensures a more consistent response compared to homogeneous balancing with $\boldsymbol{\alpha}=\operatorname{diag}\left[1,1, a^{2}\right]$.

The initial desired modal damping ratio is chosen as $\zeta_{\mathrm{des}}=$ 0.05 , corresponding to a modal mass of $\mu_{1}=0.0206$ and stiffness ratio $\widetilde{\kappa}_{1}=\mu(1-\mu)=0.0202$. When the absorber mass $m_{a}=\mu_{1} m_{1}$ has been determined, the correction parameters are obtained as $\mu_{1}^{\prime}=0.433$ and $\kappa_{1}^{\prime}=0.629$ according to the design procedure in Table 1 . After a re-tuning according to the procedure in Section 3, the final stiffness ratio becomes $\kappa_{1}=0.0199$, thus slightly smaller than the initial value.

Figure 3 shows the root loci when varying the common viscous coefficient $c_{a}$ from 0 to $\infty$. Figures $3 \mathrm{a}, \mathrm{c}$ show the root loci for absorber tuning without residual mode correction $\left(1 / \mu_{1}^{\prime}=1 / \kappa_{1}^{\prime}=0\right)$, while Figs. 3b,d show the results for the full calibration with finite correction ratios $\mu_{1}^{\prime}=0.433$ and $\kappa_{1}^{\prime}=0.629$. To illustrate the importance of a proper balancing, Figs. 3a,c represent the case with homogeneous balancing by $\boldsymbol{\alpha}=\operatorname{diag}\left[1,1, a^{2}\right]$. It is seen from Fig. $3 \mathrm{~d}$ that the full calibration with a proper balancing displays the desired semi-circular root loci with the asterisks indicating the optimal damping slightly below the bifurcation point. The red markers indicate the calibration frequencies $\omega_{A}(\circ)$ and $\omega_{B}$ ( $\square$ ). Homogeneous balancing is seen to provide limited tuning accuracy, whereas the inclusion of the residual mode correction improves the root locus diagrams in Fig. 3c,d compared to Fig. 3a,b. The modal damping ratios of the two split 
(a)

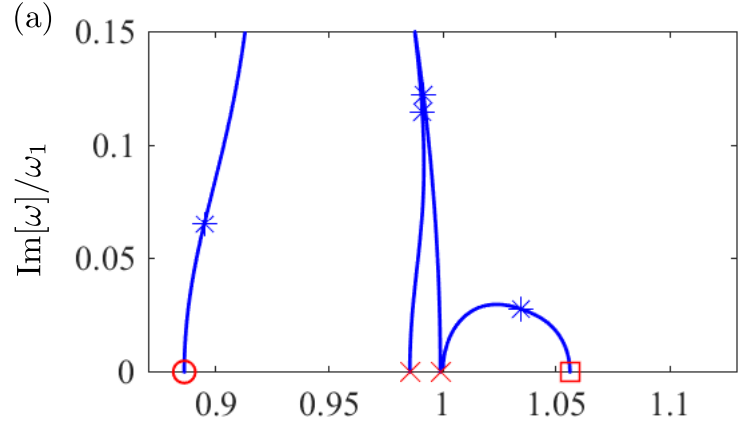

(c)

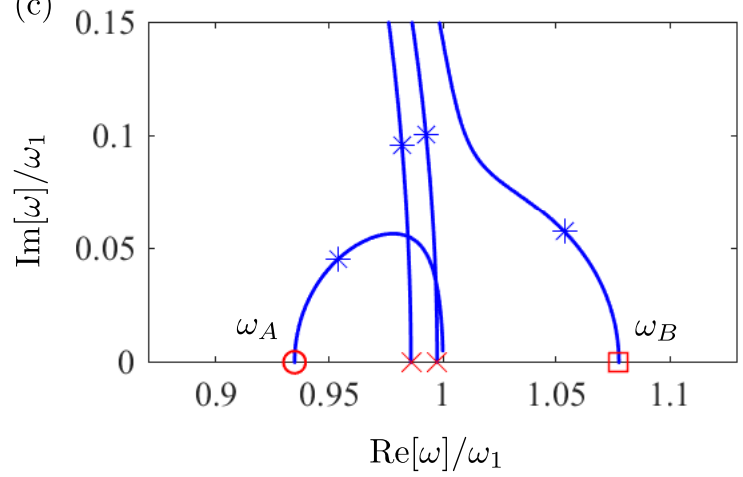

(b)

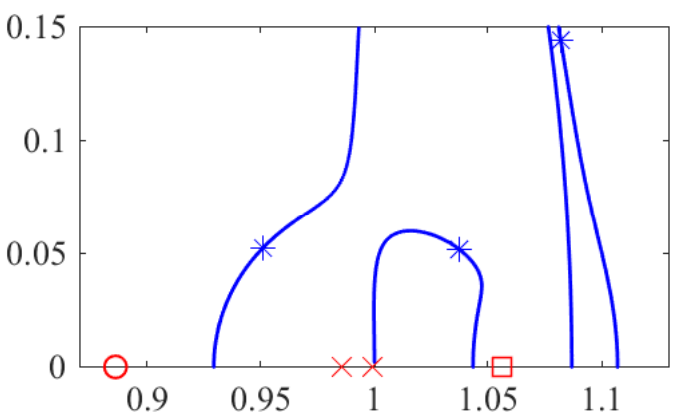

(d)

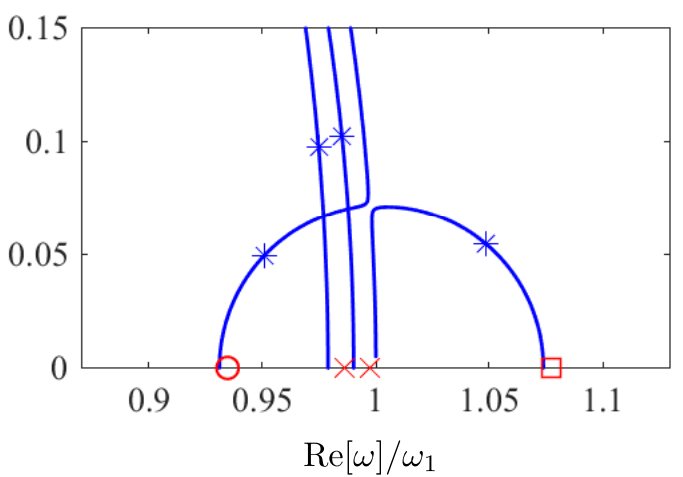

Fig. 3. Root loci $(a, c)$ without correction, $(b, d)$ with correction, $(a, b)$ not balanced and $(c, d)$ balanced.
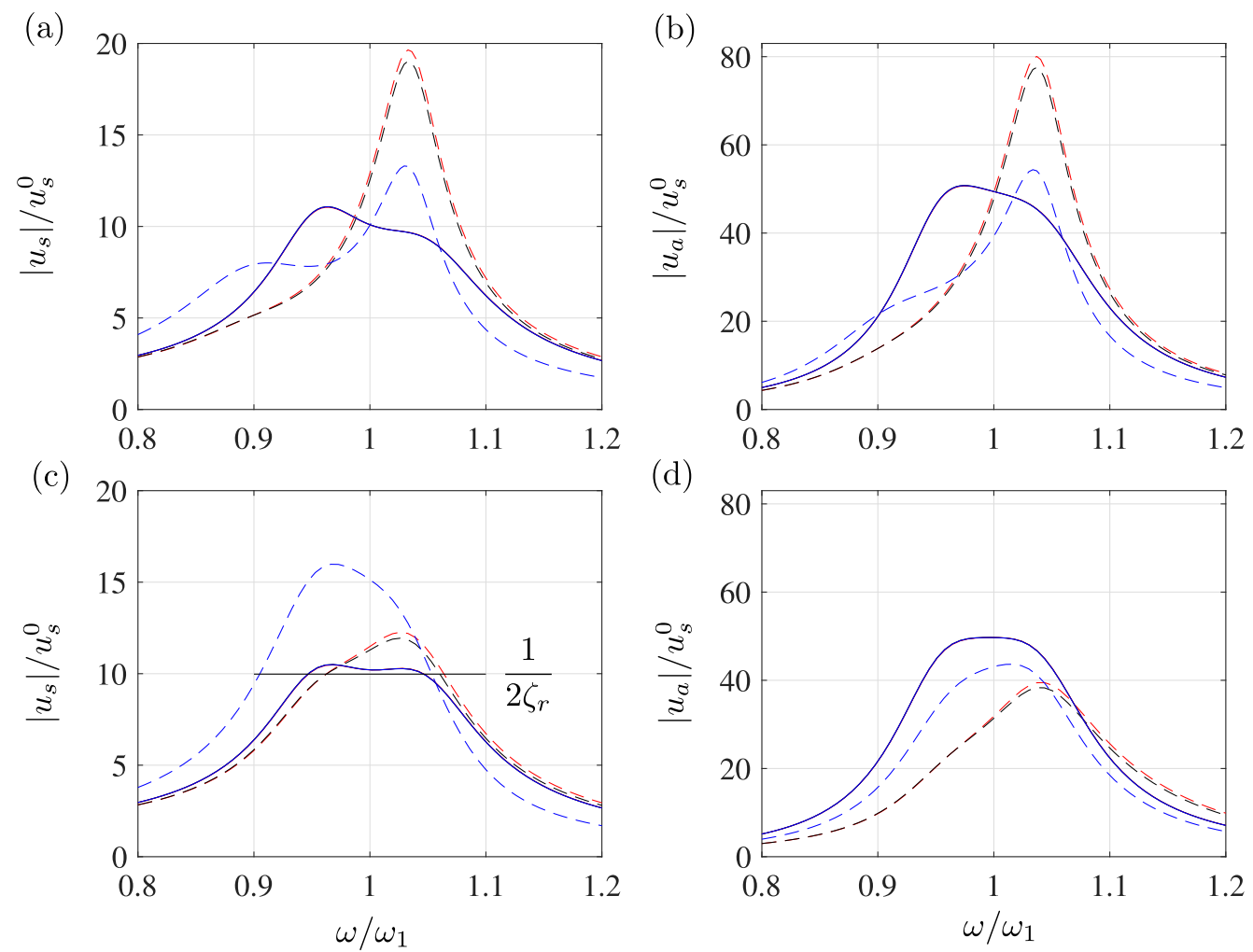

Fig. 4. Frequency response of $(a, c)$ structure, $(b, d)$ absorbers, $(a, b)$ without correction and $(c, d)$ with correction. Not balanced (dashed lines), balanced (solid lines), horizontal (red), vertical (black) and rotation (blue).

modes are according to (36) calculated to be $\zeta_{1}^{\text {est }}=0.051$, while the actual damping ratios are found to be $\zeta_{1}=0.0527$ and 0.0525 , with $\zeta_{1}=0.1005$ and 0.1043 for the two redundant absorber modes.

Figure 4 shows the frequency response curves for the beam loaded by a harmonic load with frequency $\omega$. To avoid modal interaction the spatial distribution of the load is $\mathbf{f}_{e}=\mathbf{M}_{\infty} \mathbf{q}_{\infty, 1}$, whereby it becomes orthogonal to the other modes $(j>1)$. The curves are normalized according to their corresponding static deflection of the structure $u_{s}^{0}$ at node four. Fig- 
ures $4 \mathrm{a}, \mathrm{c}$ represent the structure response, while Figs. 4b,d show the absorber amplitudes. The two top Figs. 4a,b are without residual mode calibration, while the two bottom figures $(\mathrm{c}, \mathrm{d})$ are for $\mu_{1}^{\prime}=0.433$ and $\kappa_{1}^{\prime}=0.629$. The dashed curves represent homogeneous balancing by $\boldsymbol{\alpha}=$ $\operatorname{diag}\left[1,1, a^{2}\right]$, while the solid curves represent the optimal balancing $\boldsymbol{\alpha}=\operatorname{diag}\left[1,1.735,0.038 a^{2}\right]$ with the colors indicating $u_{s}$ as the translation in the $x_{1}$-direction (red), in the $x_{2}$-direction (black) and torsion (blue). As also observed in the root locus analysis, the proper balancing of $\boldsymbol{\alpha}$ corrects the curves to coincide and exhibit the desired plateau around the resonance associated with optimal tuning of a single degreeof-freedom structure. Furthermore, the calibration ensures a flat plateau with almost identical amplification of the two structural modes in Figs. 4a,c and a completely flat plateau for the absorber amplitude in Figs. 4b,d. It is further observed that the calibration with homogeneous absorber balancing yields a significant resonance peak, illustrating the importance of proper balancing between the $\boldsymbol{\alpha}$-components by a procedure as in Section 6.

\section{Conclusions}

A procedure for approximate balancing and calibration of tuned mass absorbers mounted on flexible structures has been described. The tuning of the classic mass absorber is based on a single degree-of-freedom structure and a selected vibration mode to be damped. For discrete structures, the influence of residual non-resonant vibration modes becomes important. This contribution to the absorber tuning has been included by calibrating a flexibility and inertia term with respect to two specific frequencies identified for vanishing absorber damping and sufficiently accurate estimates of the absorber stiffness. To improve the absorber efficiency for coupled bending-torsion vibrations, a set of two translational and a single rotational absorber are combined into an effective system for damping of fully coupled vibrations. As translational and torsional stiffness are inherently different, the absorbers must not be balanced homogeneously and a method for sizing of viscous dampers has been shown to provide proper balancing for tuned mass absorbers, resulting in the desired root locus and frequency response curves with equal modal damping and flat plateaus in the corresponding response amplitude curves.

\section{References}

[1] Elias, S. and Matsagar, V., 2017," Research developments in vibration control of structures using passive tuned mass dampers", Annual Reviews in Control, vol. 44, pp. 129-156, DOI: 10.1016/j.arcontrol.2017.09.015.

[2] Arrigan, J., Pakrashi, V., Basu, B. and Nagarajaiah, S., 2011, "Control of flapwise vibrations in wind turbine blades using semi-active tuned mass dampers", Structural Control and Health Monitoring, vol. 18, pp. 840851, DOI: $10.1002 /$ stc.404.

[3] Casalotti, A., Arena, A. and Lacarbonara, W., 2014, "Mitigation of post-flutter oscillations in sus- pension bridges by hysteretic tuned mass dampers", Engineering Structures, vol. 69, pp. 62-71, DOI: 10.1016/j.engstruct.2014.03.001.

[4] Chen, S.R. and Cai, C.S., 2004, "Coupled vibration control with tuned mass damper for long-span bridges", Journal of Sound and Vibration, vol. 278, no. 1-2, pp. 449-459, DOI: 10.1016/j.jsv.2003.11.056.

[5] Zheng, L., Zhang, H., Zhao, B. and Xianjie, L., 2018, "Shaking table test and numerical simulation on vibration control effects of TMD with different mass ratios on a super high-rise structure", Structural Design of Tall and Special Buildings, vol. 27, no. 9, pp. e1470, DOI: 10.1002/tal.1470.

[6] Den Hartog, J.P., 1956, Mechanical Vibrations, 4th ed. New York, McGraw-Hill, (reprinted by Dover, New York, 1985).

[7] Krenk, S., 2005, "Frequency analysis of the tuned mass damper", Journal of Applied Mechanics, vol. 72, no. 6, pp. 936-942, DOI: 10.1115/1.2062867.

[8] Krenk, S. and Høgsberg, J., 2016, "Tuned resonant mass or inerter-based absorbers: unified calibration with quasi-dynamic flexibility and inertia correction", Proceedings of the Royal Society A - Mathematical, Physical and Engineering Sciences, vol. 472, no. 2185, pp. 20150718, DOI: 10.1098/rspa.2015.0718.

[9] Høgsberg, J., 2019, ”A robust calibration procedure for multiple electromechanical shunt absorbers on a flexible structure", Proceedings of the IX ECCOMAS Thematic Conference on Smart Structures and Materials, July.

[10] Jangid, R.S. and Datta, T.K., 1997, "Performance of multiple tuned mass dampers for torsionally coupled systems", Earthquake Engineering and Structural Dynamics, vol. 26, no. 3, pp. 307-317, DOI: 10.1002/(SICI)1096-9845(199703)26:3;307::AIDEQE639;3.3.CO;2-\#.

[11] Li, C. and Qu, W., 2006, ’Optimum properties of multiple tuned mass dampers for reduction of translational and torsional response of structures subject to ground acceleration", Engineering Structures, vol. 28, no. 4, pp. 472-494, DOI: 10.1016/j.engstruct.2005.09.003.

[12] Lin, C.C., Ueng, J.M. and Huang, T.C., 2000, "Seismic response reduction of irregular buildings using passive tuned mass dampers", Engineering Structures, vol. 22, no. 5, pp. 513-524, DOI: 10.1016/S01410296(98)00054-6.

[13] Febbo, M. and Vera, S.A., 2008, ’Optimization of a two degree of freedom system acting as a dynamic vibration absorber", Journal of Vibration and Acoustics, vol. 130, no. 1, pp. 011013, DOI: 10.1115/1.2827368.

[14] Daniel, Y. and Lavan, O., 2014, "Gradient based optimal seismic retrofitting of 3D irregular buildings using multiple tuned mass dampers", Computers and Structures, vol. 139, pp. 84-97, DOI: 10.1016/j.compstruc.2014.03.002.

[15] Zuo, H., Bi, K. and Hao, H., 2019, "Mitigation of tower and out-of-plane blade vibrations of offshore monopile wind turbines by using multiple tuned mass dampers", Structure and Infrastructure Engineering, vol. 15, no. 2, 
pp. 169-284, DOI: 10.1080/15732479.2018.1550096.

[16] Snowdon, J.C., 1984, "The cruciform dynamic vibration absorber", Journal of the Acoustical Society of America, vol. 75, no. 6, pp. 1792-1799, DOI: 10.1121/1.390980.

[17] Mokrani, B., Tian, Z., Alaluf, D., Meng, F. and Preumont, A., 2017, "Passive damping of suspension bridges using multi-degree of freedom tuned mass dampers", Engineering Structures, vol. 153, pp. 749756, DOI: 10.1016/j.engstruct.2017.10.028.

[18] Main, J. A. and Krenk, S., 2005, "Efficiency and tuning of viscous dampers on discrete systems", Journal of Sound and Vibration, vol. 286, no. 1-2, pp. 97-12, DOI: 10.1016/j.jsv.2004.09.022.

[19] Gere, J.M. and Li, Y.K., 1958, "Coupled vibrations of thin-walled beams of open cross section", Journal of Applied Mechanics, vol. A-26, pp. 373-378.

[20] Krenk, S. and Høgsberg, J., 2008, "Tuned mass absorbers on damped structures under random load", Probabilistic Engineering Mechanics, vol. 23, no. 4, pp. 408415, DOI: 10.1016/j.probengmech.2007.04.004.

[21] Krenk, S. and Høgsberg, J., 2014, "Tuned mass absorber on a flexible structure", Journal of Sound and Vibration, vol. 333, no. 6, pp. 1577-1595, DOI: 10.1016/j.jsv.2013.11.029.

[22] Krenk, S. and Høgsberg, J., 2012, 'Equal modal damping design for a family of resonant vibration control formats", Journal of Vibration and Control, vol. 19, no. 9, pp. 1294-1315, DOI: 10.1177/1077546312446796.

[23] Géradin, M. and Cardona, A., 2001, Flexible Multibody Dynamics, A Finite Element Approach, Wiley. 